\title{
Extraños en nuestras escuelas: mecanismos de discriminación educativa en Europa
}

Adrián Neubauer Esteban - Universidad Autónoma de Madrid

0000-0002-6774-1538

Recepción: 24.09.2021 | Aceptado: 16.10.2021

Correspondencia a través de ORCID: Adrián Neubauer Esteban

iD 0000-0002-6774-1538

Citar: Neubauer Esteban, A (2021). Extraños en nuestras escuelas: mecanismos de discriminación educativa en Europa. REIDOCREA, 10(36), 1-13.

Financiación: Fundación Banco Sabadell.

Resumen: El objetivo de este artículo es identificar qué mecanismos existen en los Estados miembros de la Unión Europea (UE) para limitar, o vulnerar, el derecho a la educación de la infancia inmigrante. Para ello, se realizará un metaanálisis desde el Enfoque Basado en los Derechos Humanos de estudios relacionados con la integración socioeducativa y los derechos de la infancia en estos países. Se identificaron tres dimensiones (legal, política y socioeducativa) que producen discriminación educativa hacia esta población en los países de acogida. La legal se compone dos mecanismos discriminatorios: la edad y la condición legal del menor en el país de acogida. La política consta de cuatro categorías: las políticas de austeridad; el discurso de la nueva extrema derecha europea; las políticas neoliberales en la escuela; y la existencia de campos de refugiados. En cuanto a la socioeducativa, los Estados desarrollan a menudo un modelo asimilacionista en las escuelas, algo que sumado a la escasa participación de las familias inmigrantes vulneran este derecho. A modo de conclusión, la infancia inmigrante se enfrenta a una red de mecanismos discriminatorios en la UE que dificulta su integración y el respeto de sus derechos humanos, lo que requiere de una acción conjunta por parte de toda la comunidad educativa para revertir esta situación de injusticia.

Palabra clave: Discriminación educacional

\section{Strangers in our schools: mechanisms of educational discrimination in Europe}

Abstract: The objective of this article is to identify which mechanisms of discrimination exist in the European Union (EU) to limit or vulnerate the right to education of immigrant children. For that reason, a meta-analysis was carried out from the Human Rights-based Approach of studies related to socio-educational integration and the rights of children in Member States. Three dimensions were identified (legal, political, and socioeducational) that produce educational discrimination towards this population in the host countries. First the legal dimension is made up of two discriminatory mechanisms: the age of the child and legal status of the child in the host country. Second, the political dimension has four categories: austerity policies; the discourse of the new European fair-right; neoliberal policies at school; and the existence of refugee camps in the EU. Finally, in the socio-educational dimension, States often develop an assimilationist model in schools and the low participation of immigrant families, vulnerate the full enjoyment of this right. In conclusion, immigrant children face a network of discrimination mechanisms in the EU that hinder their integration, their social advancement and respect for their fundamental rights, which requires joint action by the entire community educational to reverse this situation of injustice.

Keyword: Educational discrimination

\section{Introducción}

El nuevo milenio trajo consigo una fe renovada en el proyecto europeo, algo evidenciado en la importancia de desarrollar la ciudadanía europea en los últimos años (Valle y Matarranz, 2015). Sin embargo, dicho impulso fue una supernova, una explosión de luz, pero breve. Resulta complejo dilucidar el momento en que esa esperanza comenzó a deteriorarse, aunque si tuviéramos que elegir uno de ellos, probablemente sería el fracaso de la Constitución para Europa, un documento que tras someterse a votación no dispuso del apoyo suficiente por parte de los ciudadanos europeos (García Segura, 2019). Tras este duro revés, cuando la Unión Europea (UE) todavía trataba de cicatrizar sus heridas, la economía global sufrió una de sus mayores crisis en la historia junto al crash de 1929. 80 años más tarde el mercado financiero volvía a sentir cómo sus 
cimientos se tambaleaban por el terremoto de Lehman Brothers Holding Inc., lo que provocó un efecto dominó que afectó rápida y abruptamente a las economías europeas (García Ruiz, 2015). Como consecuencia de ello los Estados miembros entraron en recesión, se redujo la inversión y el consumo, la tasa de desempleo se elevó exponencialmente y el sector público fue incapaz de garantizar los servicios básicos (educación, sanidad...) con solvencia (García Ruiz, 2015).

Ante esta situación de colapso, las autoridades europeas decidieron intervenir las economías de los Estados miembros que se vieron más afectados, siendo una parte considerable de ellos los del sur de Europa (Grecia, Italia y Portugal) (González y Guillén, 2018). La postura adoptada por la UE fue la de implementar políticas de austeridad, lo que aumentó considerablemente la precariedad de sus servicios sociales (López Pereira, 2018). Todo ello acentuó la fractura entre la ciudadanía nacional y las instituciones europeas, quienes fueron vistas como las causantes de los males del pueblo (Pou, 2018).

Tras unos duros años de recuperación, la economía europea vislumbró algunos brotes verdes, pero en el horizonte se atisbaba un nuevo incendio que el proyecto europeo debía apagar conjuntamente, o bien, sería reducido a cenizas: la crisis de refugiados. Desde el año 2014 al 2016 la UE recibió más de 3 millones de solicitudes de asilo, siendo aproximadamente un tercio de los niños (Eurostat, 2020). La llegada de estas personas ha sido mayoritariamente a los países del sur de Europa, que como mencionábamos con anterioridad, fueron de los más afectados por la crisis económica de 2008, por lo que han tenido que hacer frente a este fenómeno demográfico con unos servicios sociales muy mermados e insuficientes (González y Guillén, 2018), lo que ha llevado incluso a vulnerar los derechos humanos de los solicitantes de asilo. FerreroTurrión (2016, p. 171) critica a la ciudadanía europea por ser "insensible a los dramas más allá de sus fronteras", lo que inevitablemente nos urge a repensar los valores de la UE y su compromiso con los derechos humanos.

La crisis de refugiados ha acentuado un sentimiento de inseguridad, incertidumbre y desprotección frente a la globalización, que se presenta como una amenaza para la identidad europea (Sanahuja, 2016), un hecho que se acentúa con la libertad de movilidad en el seno de la UE (Campani, 2019). La gestión por parte de la UE y de sus Estados miembros ha sido deficitaria. Por un lado, las políticas de integración han creado tensiones y problemas (León y Subirats, 2015). Además, se ha instaurado la idea colectiva de que la estabilidad de las democracias liberales y estados del bienestar europeos requieren la exclusión de otros, lo que cuestiona la legitimidad del Estado y del proyecto europeo (Aris, 2016).

La actuación de los Estados miembros ha dejado en evidencia la política migratoria común de la UE, pues si por algo se ha caracterizado es por su insolidaridad (FerreroTurrión, 2016). Esta cuestión se refleja en que el reparto para el reasentamiento de los solicitantes de asilo en los diferentes Estados no se ha respetado, ya que Polonia, República Checa y Hungría se han negado a aceptar su cupo (Eurostat, 2020). Por otro lado, la dinámica en la aceptación de solicitudes de asilo ha variado, pues mientras Suecia fue un ejemplo en los primeros años en la acogida de estas personas, en 2019 apenas aceptó el 29,3\% de dichas solicitudes en primera instancia (Eurostat, 2020). Asimismo, España se ha elevado hasta la primera posición aceptando el 66,2\% de ellas, mientras que a principios de la crisis su posicionamiento era bien distinto (Eurostat, 2020).

Este cocktail geopolítico ha hecho resurgir movimientos xenófobos y antieuropeos en el seno de la UE, que ha sido cristalizado en los cargos que desempeñan políticos afines 
a la nueva extrema derecha en las instituciones democráticas nacionales y europeas (Parlamento Europeo, 2019). El Brexit es un proceso sociopolítico que ha surgido a raíz del desencanto de la ciudadanía británica con la UE, quien fue usada como chivo expiatorio por los políticos del Vote Leave para culparle de la migración, el desempleo y los recortes en servicios sociales que padece la nación británica (Rubiés, 2019).

Dicho esto, es fundamental identificar los mecanismos de discriminación socioeducativa que se están produciendo en los Estados miembros, qué fenómenos y agentes influyen en ellos, para posteriormente elaborar propuestas de intervención acorde a los derechos humanos y los valores radicales de la UE.

\section{Marco internacional contra la discriminación}

La legislación internacional en materia de derechos se rige bajo cuatro principios fundamentales (De Beco, 2009): derecho a la educación, derecho a la seguridad, derecho a la participación y la no-discriminación. Como bien indica Rabossi (1990), es precisamente en este ámbito donde inicialmente se regula y desarrolla el principio de igualdad o el de no-discriminación. Por ese motivo, en este apartado sintetizaremos brevemente dichos referentes legales y cómo abordan esta cuestión, especialmente en el caso de la población migrante.

Asimismo, el primer documento es la Declaración Universal de los Derechos Humanos (1948, Art. 7), donde se establece que "todos son iguales ante la ley y tienen, sin distinción, derecho a igual protección de la ley. Todos tienen derecho a igual protección contra toda discriminación que infrinja esta Declaración y contra toda provocación a tal discriminación".

Más tarde surgió la Declaración de los Derechos del Niño (1959), que establece "el derecho a la igualdad, sin distinción de raza, religión o nacionalidad" en su primer principio. Posteriormente se desarrollaron algunas convenciones específicamente destinadas a combatir la discriminación racial (Convención Internacional sobre la Eliminación de todas las Formas de Discriminación Racial, 1965) y contra la mujer (Convención sobre la eliminación de todas las formas de discriminación contra la mujer, 1979). Sobre los derechos de la infancia, en 1989 la ONU ratificó la Convención sobre los Derechos del Niño, por la cual los Estados han de garantizar la no-discriminación de la infancia migrante (Art. 2).

En el derecho europeo el principal referente es la Carta de los Derechos Fundamentales de la UE (DO, C202, 2016). El artículo 14 regula el derecho a la educación y garantiza la educación obligatoria y gratuita a toda persona. Además, el artículo 21 prohíbe cualquier tipo de discriminación.

Poniendo el foco en la población migrante, en los últimos años ha habido un auge en el desarrollo de legislación internacional para garantizar sus derechos fundamentales. La Declaración de Nueva York para los Refugiados y los Migrantes (2016) refuerza el derecho a la igualdad de trato y oportunidades de esta población, protegiéndolos especialmente de las redes de explotación, siendo esto urgente en el caso de las niñas y las mujeres. Para ello, invita a los Estados a adoptar "medidas para mejorar su integración e inclusión, según proceda, teniendo en cuenta en particular el acceso a la educación, la atención de la salud, la justicia y la enseñanza de idiomas" (Declaración de Nueva York, 2016, p. 4), por lo que la educación cobra un papel clave en la erradicación de la xenofobia y en la lucha por los derechos humanos. Finalmente, el Pacto Mundial para la Migración Segura, Ordenada y Regular (2018) consolida lo expuesto en la Declaración de Nueva York (2016) con relación a la implementación de 
medidas preventivas y compensatorias para reducir la vulnerabilidad de esta población (Art. 7). No obstante, Rabossi (1990) advierte que estas cláusulas son abiertas, pues si no lo fueran dejarían abierta la posibilidad de llevar a cabo otros tipos de discriminación no recogidos en dichos referentes legales.

\section{Discriminación: conceptualización, causas y repercusiones}

Tras haber presentado el marco internacional contra la discriminación, es preciso reflexionar detenidamente sobre el concepto de discriminación, las causas que producen este fenómeno desde una perspectiva social e individual y sus consecuencias. Inicialmente debemos señalar que, aunque la legislación internacional en materia de derechos humanos utilice recurrentemente el principio de no-discriminación, su conceptualización puede ser entendida sociopolíticamente desde perspectivas muy diferentes, por lo que una primera conclusión sobre este término es que es polisémico (Herzog, 2011). En esta investigación concluimos que la discriminación es el conjunto de prácticas institucionales, sociales e individuales que niegan la igualdad de oportunidades y de trato a colectivos por sus condiciones particulares, atentando contra la justicia social mediante la negación de sus derechos fundamentales (Rabossi, 1990; Prevert et al., 2012; Solís, 2017).

Dichas prácticas a menudo tienen lugar como consecuencia de la estructura y el orden social existente, que impide, o niega, el reconocimiento de ciertas colectividades (Prevert et al., 2012; Solís, 2017). Para que esta coyuntura se produzca debe haber unos agentes sociales que dispongan de la legitimidad y el poder suficiente para llevarlas a cabo. Estos actores atribuyen a estos colectivos una posición de inferioridad, lo que legitima vulneraciones de derechos por no considerarlos merecedores de ellos (Prevert et al., 2012).

Estas prácticas discriminatorias permean en todas las esferas de la vida pública y privada, pues están fuertemente influenciados por la económica, la historia, la cultura y la política (Solís, 2017). Por ello, su influencia es notable, de modo que debe ser atendida desde una perspectiva sistémica. Una consecuencia es la aceptación por parte de los colectivos oprimidos de su situación, llegando incluso a culpabilizarse a sí mismos de su desventaja social (Prevert et al., 2012). En segundo término, estas personas padecen cuadros psicoemocionales realmente delicados, principalmente asociados con el estrés, la ansiedad y la depresión (Prevert et al., 2012). Por si fuera poco, la discriminación es un fenómeno acumulativo, que persiste a lo largo de la vida de los afectados y de sus familiares, incluso durante generaciones (Solís, 2017).

En definitiva, el conjunto sistemático y sostenido de estas prácticas, legitimado por los prejuicios e intereses particulares de los grupos dominantes, perpetúa su posición social privilegiada a costa de los derechos fundamentales de los grupos más vulnerables, como es el caso de los migrantes, solicitantes de asilo y refugiados.

Centrándonos en estos colectivos, las prácticas explicadas anteriormente les excluyen de la sociedad de primera categoría, convirtiéndolos en ciudadanos de segunda clase. Herzog (2011) afirma que este fenómeno se debe principalmente a su difícil situación socioeconómica, que les impide afrontar el pago de las actividades socioculturales que ofrece la comunidad, limitando su libertad y derecho a participar activamente en ella. En el ámbito político también existen prácticas que vetan su implicación, como es la negación del derecho a voto, siendo este un derecho fundamental en democracia (Herzog, 2011). Sin embargo, el caso más extremo es el de aquellos que son expulsados del país por no adquirir la ciudadanía o el permiso de residencia, quienes física y simbólicamente son desechados por la sociedad de acogida (Herzog, 2011). 
Pese a que históricamente han tenido lugar acontecimientos, políticas y discursos antinmigrantes, en los últimos años estos fenómenos han vivido un repunte considerable como consecuencia de lo expuesto en el primer apartado del artículo, las crisis económicas, políticas y migratorias. Como introdujimos anteriormente, la nueva extrema derecha ha hecho uso de este contexto para sacar rédito electoral. Así, es importante poner de manifiesto dos de sus estrategias clave que explican su ascenso electoral: el discurso del miedo y la construcción social de la otredad.

Estos partidos ofrecen una visión distorsionada y negativa de la población inmigrante, cuyos efectos en la sociedad de acogida, o en nuestro pueblo, hacen temblar nuestros cimientos. Una de las principales cuestiones de las que culpabilizan a la población inmigrante es la precariedad y la ausencia de ayudas sociales (López Ortega, 2017). Por otro lado, el pueblo de acogida siente la constante amenaza de ser desprovista de su identidad cultural propia (López Ortega, 2017). En este escenario, la población musulmana se erige como la principal amenaza ante nuestra forma de vida occidental (Astier y Errasti, 2018). Además, los medios de comunicación juegan un papel determinante en la construcción social de la población inmigrante, provocando que la sociedad de acogida desarrolle una cultura del miedo hacia lo extraño (López Ortega, 2017).

A modo de síntesis, estos partidos políticos, las clases dominantes y los medios de comunicación contribuyen profundamente a que la sociedad de acogida desarrolle una imagen sesgada de la población inmigrante, lo que condiciona significativamente las formas de interacción entre ambos colectivos (Izaola y Zubero, 2015). Como consecuencia de ello la sociedad de acogida europea desarrolla, o perpetúa, estereotipos xenófobos hacia esta población, legitimándose a menudo en la protección y la seguridad de nuestra cultura, nuestra forma de vida y la de nuestros hijos.

\section{Metodología}

\section{Definición del problema y objetivos}

La crisis económica de 2008, el Brexit, el auge de la nueva extrema derecha y la crisis de refugiados de 2015 han aumentado notablemente la incertidumbre para garantizar el derecho a la educación de la población inmigrante en la UE. Esta cuestión se torna fundamental en la integración de estas personas en la sociedad europea, por lo que esta investigación tiene como objetivo identificar qué mecanismos existen en los Estados miembros de la UE para limitar, o vulnerar, el derecho a la educación de la población inmigrante. Responder a esta pregunta permitiría conocer la realidad a la que se enfrenta esa población y a su vez realizar una serie de propuestas para hacer frente a los mecanismos discriminatorios hallados, lo que se corresponde con el segundo objetivo del estudio.

\section{Método}

El Enfoque Basado en los Derechos Humanos (EBDH) nos permite realizar una aproximación multidisciplinar y sistémica de esta problemática a partir de la legislación internacional en materia de derechos humanos, para posteriormente elaborar propuestas de mejora con base en dichos referentes legales (UNICEF, 2008; Neubauer, 2020). Este método se compone de cuatro fases: (i) evaluación y análisis de situación; (ii) evaluación de la capacidad de ejecución; (iii) planificación, diseño y ejecución de los programas; (iv) supervisión y evaluación. 
La fase inicial consiste en llevar a cabo una evaluación y un análisis de la situación actual, es decir, de qué modo y quiénes están vulnerando los derechos humanos, pues debemos recordar que estos son interdependientes, tal y como se recoge en el artículo 28 de la Declaración Universal de los Derechos Humanos (Otero et al.,2016). Con el fin de que este análisis se realice con la mayor rigurosidad y exhaustividad posible es preciso partir de la legislación internacional en materia de derechos humanos como referente. En el caso de que se estén evaluando los derechos de un colectivo particular, también es esencial tener en cuenta el marco jurídico internacional que garantiza sus derechos fundamentales. Dado que este artículo pone el foco en la discriminación que padece la población inmigrante, deberemos partir de los documentos normativos presentados en la introducción.

Una vez realizada la evaluación inicial, es el momento de evaluar la capacidad de ejecución de los actores implicados. Dentro de los actores debemos diferenciar dos tipos: los agentes cuyos derechos están siendo vulnerados y las autoridades. Si bien todos ellos tienen una capacidad de reivindicación y denuncia limitada, esto se acentúa en las víctimas. Por ese motivo, en esta fase se ha de determinar su potencial para defender sus derechos y exigir a los agentes responsables que los garanticen acorde a la legislación internacional en materia de derechos humanos. En segundo lugar, se encuentran las autoridades competentes, que pueden abarcar desde las locales a las supranacionales, cuya capacidad de actuación, a priori, es mayor que la de las víctimas. Sin embargo, a menudo los agentes implicados tienen intereses encontrados, lo que se traduce en tensión y falta de cooperación, por lo que estas fricciones se han de hacer explícitas en esta fase, al igual que los puntos compartidos.

A raíz de ello se podrán planificar, diseñar y poner en marcha diferentes programas para revertir las situaciones de injusticia y garantizar los derechos fundamentales de las personas afectadas. Durante estos procesos de planificación, diseño y ejecución deben participar activamente todos los actores involucrados: las autoridades, la comunidad, la sociedad civil y los titulares de derechos. De este modo, las medidas adoptadas satisfarán a todas las partes, otorgando a los programas de una mayor sostenibilidad gracias al respaldo comunitario y político. Finalmente, se han de desarrollar una serie de mecanismos, procesos e instrumentos para monitorizar el cumplimiento de los programas implementados, debiendo, una vez más, involucrarse toda la comunidad en el diseño, la implementación y el proceso de evaluación.

\section{Recogida de datos e instrumento de análisis}

En primer lugar, se realizará metaanálisis de estudios e investigaciones cuyo objeto de estudio sea la integración socioeducativa o los derechos de la infancia en los Estados miembros desde el EBDH. Como indicamos anteriormente en el apartado metodológico, el EBDH requiere evaluar y analizar la situación actual de nuestro objeto de estudio, en nuestro caso de qué modo la población inmigrante, solicitante de asilo y refugiada. Una vez hecho esto, se han identificado tres dimensiones, una de carácter legal, otra política y en último lugar la socioeducativa. La primera de ellas está conformada por dos mecanismos que emplean los Estados para limitar el derecho de la infancia migrante: la edad y su condición de ilegalidad en el país de acogida. Más tarde, la dimensión política dispone de cuatro categorías diferentes: las políticas de austeridad; el discurso de la nueva extrema derecha europea; las políticas neoliberales en la escuela; y la existencia de campos de refugiados en la UE. En último lugar, los Estados desarrollan a menudo un modelo asimilacionista en las escuelas, algo que sumado a la escasa participación de las familias inmigrantes vulneran el pleno disfrute de este derecho desde una perspectiva socioeducativa, que es nuestra tercera dimensión. 
Tabla 1: Matriz de análisis de los mecanismos de discriminación

\begin{tabular}{ll}
\hline Dimensión & Categoría \\
\hline \multirow{2}{*}{ Legal } & Edad \\
\cline { 2 - 2 } & Condición de ilegalidad \\
\hline Política & Políticas de austeridad \\
\cline { 2 - 2 } & Nueva extrema derecha europea \\
\cline { 2 - 2 } & Neoliberalismo \\
\cline { 2 - 2 } & Campos de refugiados \\
\hline Socioeducativa & Modelo asimilacionista \\
\cline { 2 - 2 } & Escasa participación de las familias inmigrantes \\
\hline
\end{tabular}

\section{Resultados}

\subsection{Mecanismos de discriminación socioeducativa hacia la población inmigrante en la UE}

\section{Mecanismos legales de discriminación}

Para comenzar, es preciso reiterar que los Estados tienen la obligación de garantizar una educación obligatoria, gratuita y accesible a todo menor bajo el principio de nodiscriminación (Convención sobre los Derechos del Niño, 1989), pero dicha responsabilidad expira una vez estos niños alcanzan una edad superior a la de escolarización obligatoria estipulada en dicho país.

Actualmente solo Alemania, Austria, Bélgica, Francia, Polonia y Portugal establecen el final de la educación obligatoria a los 18 años (Eurydice, 2020). El modelo más recurrente es el de fijarla a los 16 años, ya que 15 Estados miembros abogan por ello, siendo algunos de ellos Dinamarca, Letonia o Finlandia (Eurydice, 2020). En un término medio se sitúa Rumanía, que la establece a los 17 años (Eurydice, 2020). En el otro extremo están Chipre, Croacia, Grecia, República Checa y Eslovenia, que fijan el final de la escolarización obligatoria a los 15 años (Eurydice, 2020).

El resultado de ello es que a excepción de los Estados cuya enseñanza obligatoria abarca hasta los 18 años, los demás están exentos de garantizar una escolarización a los menores inmigrantes recién llegados cuyas edades sean superiores a las marcadas por la legislación nacional, como es el caso de 21 Estados miembros, es decir, en el $77,77 \%$ de toda la UE.

Por otro lado, algunos Estados se escudan en la condición de ilegalidad del menor para negarle o brindarle un trato diferenciado en el ámbito educativo (Nilsson y Bunar, 2016; Grabbe, 2018). En el caso de Alemania es complejo hacer una aproximación exhaustiva sobre esta cuestión debido a la gran autonomía de la que disponen los Länder. Un ejemplo de ello es que el derecho a la educación de los inmigrantes ilegales y de la infancia refugiada puede recogerse de forma muy desigual a lo largo del territorio nacional, siendo este más explícito en alguno Länder que en otros, debido principalmente a que no existe un referente nacional que regule este derecho fundamental de forma rotunda y nítida (Grabbe, 2018). Siguiendo con Alemania, estos menores no pueden ejercer de aprendices en el sistema educativo, lo que supone un trato desigual hacia este colectivo (Nilsson y Bunar, 2016).

Algo similar sucede con Suecia, puesto que los municipios son quienes deben regular el derecho a la educación de la infancia solicitante de asilo. Sin embargo, la peculiaridad del Estado Sueco es que estos menores no tienen la obligación de acudir a la escuela a pesar de estar en edad de escolarización, quedando a su voluntad el participar en las 
actividades educativas y formativas ofertadas por el país de acogida (Nilsson y Bunar, 2016).

Asimismo, algunos Estados disponen de una normativa que estipula el tiempo máximo para matricular en el sistema educativo al alumnado inmigrante de nueva incorporación, pero estos pueden variar, o existir, en función del estatus legal del menor. Este es el caso de la infancia refugiada en Estonia y Chipre, o bien de los que hayan solicitado asilo formalmente en Grecia, Rumanía, Eslovenia y Eslovaquia (Eurydice, 2019). El caso más llamativo es el de Lituania, que fija en 30 días este plazo para los que hayan obtenido el permiso de residencia, mientras que este periodo se triplica para los solicitantes de asilo (Eurydice, 2019).

\section{Mecanismos políticos de discriminación}

Como resultado de las medidas de austeridad adoptadas por la UE, los Estados miembros más afectados por la crisis afrontaron fuertes recortes en sus servicios sociales y programas de atención a los sectores vulnerables, por lo que desde entonces la inversión es escasa e ineficaz (Koehler, 2017), que fue acentuado en países del sur como Grecia e Italia.

Este fenómeno se relaciona estrechamente con el auge de la nueva extrema derecha europea, quien como abordamos con anterioridad, consolida el discurso de ellos contra nosotros, ampliando la brecha y la desconfianza entre la comunidad de acogida y la inmigrante (Izaola y Zubero, 2015). Como resultado, existe un riesgo potencial de que si estos partidos mejoran sus resultados electorales en los próximos años se implementen nuevas medidas a favor de la ciudadanía nacional, excluyendo así paulatinamente a la población inmigrante de la vida pública en el país de acogida.

Otras políticas que perpetúan la discriminación de la población inmigrante son las políticas de corte neoliberal en el ámbito educativo, siendo su máximo exponente la libertad de elección. Esta medida ha sido criticada por favorecer la segregación escolar del alumnado migrante (Reath, 2013). Ello se produce por el fuerte desconocimiento de las familias inmigrantes sobre el sistema educativo de acogida y la negativa de la población nativa a que sus hijos estudien en centros con un elevado porcentaje de alumnado extranjero. De hecho, familias autóctonas de clase media se mudan a otras zonas residenciales donde hay menor población inmigrante (Rahona y Morales, 2013). Este fenómeno ahonda en las desigualdades estructurales y sociales que padece la población inmigrante, quien lleva una vida paralela a la sociedad de acogida (Adam, 2015).

No obstante, esta desigualdad de oportunidades, desarrollar una vida paralela, la segregación residencial y escolar alcanzan su máximo exponente en los 26 campos de refugiados existentes en la UE, donde habitualmente la educación que reciben estos menores, si es que la reciben, es aislada, limitada y de baja calidad (Neubauer, 2020).

\section{Mecanismos socioeducativos de discriminación}

Los mecanismos de discriminación socioeducativa se reflejan de dos modos. Diferentes investigaciones apuntan (Ocampo y Cid, 2012; Edling y Francia, 2017) a que los Estados tienden a promover un modelo asimilacionista en los centros educativos. Por lo tanto, los estudiantes inmigrantes y sus familias se ven empujados a adoptar la cultura y los valores del país de acogida, lo que genera tensión entre los centros escolares y las familias, e incluso entre el alumnado y sus familias (Corchia, 2015). Dicho esto, los programas de educación intercultural, ya de por sí insuficientes en algunos países como 
Italia (Modesti, 2011), no han permeado en los sistemas educativos como debieran por la escasa formación del profesorado en esta materia (Santagasti, 2016). Dicho esto, podemos concluir que hasta la efectividad de los programas interculturales ha quedado en entredicho.

Finalmente, las familias inmigrantes tienen una escasa participación en la vida escolar de sus hijos por varios factores. La principal barrera que se encuentran es la lingüística, lo que les impide comunicarse con solvencia con la institución educativa (Etxeberria y Elosegui, 2010). Otro elemento importante es el desconocimiento del sistema educativo de acogida por parte de las familias inmigrantes (Bini, 2017). Por otro lado, algunos docentes adoptan una posición de rechazo a estas familias, especialmente en el caso de las musulmanas (Ahmadi et al., 2020). Esta desconfianza y desentendimiento mutuo cristaliza en reproches mutuos (Soriano-Ayala y González-Jiménez, 2010), lo que distancia a las instituciones educativas de las familias y viceversa.

\subsection{Diálogo entre actores y sus intereses particulares}

Para comenzar, es preciso identificar los titulares de derechos, es decir, quiénes son las personas o colectivos que están viendo vulnerado su derecho a la educación. Dicho esto, en nuestro estudio los titulares son todos los menores de 18 años (Convención sobre los Derechos del Niño, 1989) que sean migrantes (legales e ilegales), incluyendo a los solicitantes de asilo, refugiados, apátridas, indocumentados y menores no acompañados.

Los responsables de garantizar este derecho son la UE y los Estados miembros. Por un lado, este organismo supranacional orienta las políticas monetarias, económicas, sociales y educativas de los Estados miembros, de modo que la línea que establezca tendrá una trascendencia notable en esta cuestión. No obstante, el principal responsable es el Estado, pues acorde a la legislación internacional en materia de derechos humanos, es quien debe asegurar una educación de calidad, gratuita, obligatoria y universal a todo menor (Convención sobre los Derechos del Niño, 1989).

Tras identificar a los titulares de derechos y a los responsables de garantizarlos, es el momento de poner en diálogo a los actores implicados, de modo que arrojemos luz sobre sus intereses y cómo estos chocan o convergen entre sí a partir de las dimensiones y mecanismos de discriminación expuestos anteriormente.

\section{Dimensión legal}

El Estado dispone de una serie de intereses diferentes a las familias inmigrantes y a los propios menores. El hecho de que el Estado no garantice el derecho a la educación a los niños hasta los 18 años merma las altas expectativas de las familias inmigrantes de que sus hijos asciendan socialmente (Baltaci, 2017). Así, se perpetúa una desigualdad de oportunidades educativas y económicas, lo que también podría ir en contra de los intereses de las empresas, quienes ven considerablemente limitada la oferta de trabajadores por esta cuestión.

En este sentido las familias autóctonas pueden posicionarse a favor de esta medida y la de poner barreras en el acceso a aquellos menores indocumentados o en situación ilegal. Esta postura podría explicarse por la limitada oferta de plazas formativas de la que disponen los Estados, especialmente en la Formación Profesional, de modo que estas familias defienden los intereses particulares de sus hijos frente a los extraños. Dicha argumentación también es empleada por la nueva extrema derecha, quien considera que los recursos disponibles del Estado han de ponerse a disposición del 
pueblo nacional, especialmente si son escasos como sucedió en la crisis de 2008 y corre el riesgo de ocurrir tras el coronavirus.

\section{Dimensión política}

A raíz de la crisis económica de 2008 y de refugiados de 2015 la nueva extrema derecha vivió un auge notable en la UE. Por lo tanto, podemos deducir que aquellas personas que se vieron más afectadas por ambas crisis, es decir, aquellos que hayan perdido sus empleos o empeorado su calidad de vida, tienen cierta predisposición a apoyar el discurso de estos partidos políticos y culpabilizar a la UE y a los inmigrantes de su situación. Sin embargo, las políticas de austeridad implementadas por este organismo supranacional también han causado estragos en asociaciones de inmigrantes, organizaciones no gubernamentales y en los servicios sociales nacionales, así que en cierto modo todos los agentes, a excepción de la UE, son contrarios a estos recortes económicos.

Con relación a las políticas neoliberales debemos destacar las posturas opuestas de las familias inmigrantes y las nativas, dado que estas últimas no son partidarias de que sus hijos estudien en centros con un elevado porcentaje de población inmigrante (Rahona y Morales, 2013). Por lo tanto, la primera convergencia que encontramos es la de estas familias autóctonas y la nueva extrema derecha, que a menudo defiende la libertad de elección como un derecho de las familias en el marco del derecho a la educación. No obstante, debemos señalar que la UE ha reconocido que una de las principales barreras para hacer plenamente efectivo el derecho a la educación es la segregación escolar (DO, C378/1, 2013), por lo que sus intereses son compartidos por los estudiantes y las familias inmigrantes.

Sobre los campos de refugiados, la UE converge con los partidos de la nueva extrema derecha al legitimar el rechazo de un considerable número de solicitudes de asilo en defensa de la seguridad nacional, una idea contraria a la de las ONG y los solicitantes de asilo. Sin embargo, es preciso apuntar que el posicionamiento de la UE puede variar en función del referente legal que consultemos, pues este organismo también se encuentra en una disputa interna entre sus intereses político-económicos y sus valores intrínsecos (dignidad humana, derechos humanos, solidaridad...). Asimismo, debemos tener en cuenta la advertencia de Payero (2019), quien apunta que la principal preocupación de la UE en esta cuestión está siendo asegurar el equilibrio y el buen funcionamiento del mercado interior, lo que podría contradecir sus valores originarios.

\section{Dimensión socioeducativa}

Sobre el modelo asimilacionista, podemos concluir que el Estado de acogida tiene el deseo de que la población inmigrante adquiera sus valores, sus costumbres, su cultura y su lengua, algo que para la nueva extrema derecha es innegociable. Este afán contradice la línea establecida por la UE de fomentar la educación intercultural en los centros (DO, L77/44, 2014), una idea secundada por las asociaciones de inmigrantes, las ONG, los estudiantes y las familias inmigrantes.

\section{Discusión}

Una vez analizada la situación, la capacidad de los agentes implicados para reivindicar sus derechos fundamentales y expuesta la interrelación de los intereses de cada colectivo, es el momento de plantear algunas propuestas de intervención (UNICEF, 2008; Neubauer, 2020). Con relación a la dimensión jurídica, los Estados miembros deberían involucrar a toda la comunidad educativa en el diseño, la implementación y la 
evaluación de las políticas migratorias, sociales y educativas. La participación activa de ONG, de expertos en la materia y especialmente de los titulares de derechos, en este caso la infancia inmigrante, permitiría ofrecer una respuesta adecuada a las necesidades particulares de este colectivo. Por otro lado, dichos referentes normativos han de fundamentarse desde la dignidad humana, de modo que ningún menor sea excluido de la educación por cuestiones legales o etarias, garantizando así un trato igualitario a toda persona (Pelé, 2004).

Este hecho requiere una actuación urgente por parte de los Estados miembros, ya que a menudo se producen contradicciones jurídicas entre las leyes migratorias y educativas (Flores, 2018). Dicho conflicto tiende resolver favorablemente a favor de la legislación en materia de inmigración, que son responsabilidad del Estado, lo que provoca tensiones entre la libertad de las autoridades regionales y locales en materia educativa. Asimismo, siempre debería primar la condición de menor sobre la de inmigrante, ya que según la Convención sobre los Derechos del Niño (1989) el Estado debe asegurar una educación de calidad, gratuita, obligatoria y universal a todo menor, lo que pone de manifiesto el conflicto entre las normativas supranacionales, nacionales, regionales y locales con respecto al acceso y al derecho a la educación de los menores inmigrantes en situación irregular.

Dicho esto, una primera iniciativa que deberían plantearse los Estados es la de extender la educación obligatoria hasta al menos los 18 años. Sin embargo, es cierto que esto iría de la mano de una mayor inversión en educación, lo que para algunos Estados resultaría complicado. Por ese motivo, un modelo óptimo podría ser el que plantean Austria y Polonia, quienes estipulan un periodo de educación obligatoria desde los $15 \mathrm{a}$ los 18 años a tiempo parcial (Eurydice, 2020). Ello permitiría a los Estados reducir la inversión en esta etapa y al mismo tiempo favorecer la inclusión laboral de los menores que así lo deseen, ya sea mediante trabajo remunerado o prácticas.

Aunando las dimensiones política y socioeducativa, en primer lugar, la UE no debe repetir los errores cometidos en la crisis de 2008, donde las políticas de austeridad asfixiaron a los servicios sociales de los países más afectados (Horvat y Žižek, 2014). Con vistas al panorama venidero a raíz de la crisis del coronavirus, se deberían promover iniciativas político-económicas más flexibles que promuevan la inversión en estos países. No obstante, no debemos perder de vista que la educación genera beneficios económicos a medio plazo, por lo que invertir en ella se hace más necesario que nunca.

Con respecto al auge de la nueva extrema derecha, el papel que cobran las instituciones educativas en esta cuestión es trascendental. Desde ellas es urgente que se desarrollen actividades que promuevan la educación intercultural. Para ello, sería conveniente que participara activamente toda la comunidad educativa (expertos, ONG, asociaciones de vecinos...) y las familias inmigrantes, quienes de este modo se involucrarían más en la vida escolar de sus descendientes.

Del mismo modo, los estudiantes han de adquirir un pensamiento crítico sólido, ya que la nueva extrema derecha se apoya en los medios de comunicación para fomentar el rechazo a la comunidad inmigrante, habitualmente mediante fake news y el discurso del miedo. Algunas propuestas pedagógicas óptimas para desarrollar este enfoque educativo sería el aprendizaje por proyectos y el Aprendizaje Servicio, pues ambos favorecen el pensamiento crítico y están dotados de una dimensión comunitaria (Puig et al., 2011). 
Una consecuencia de estos programas interculturales sería el desarrollo de un tejido social en el que la comunidad inmigrante se sienta aceptada, respetada y valorada por la de acogida. Además, las familias nativas serían menos reticentes a que sus hijos estudiaran en centros con un alto índice de población inmigrante, por lo que los estragos de la libertad de elección se reducirían en términos de segregación escolar y residencial.

Por otro lado, esta investigación cuenta con una serie de limitaciones que es preciso señalar. En primer lugar, la revisión realizada no es sistemática. Además, el estudio no presenta detalladamente el estado de la cuestión en toda la UE, pero sí ofrece una panorámica global de esta. Asimismo, de cara al futuro sería interesante replicar el estudio en otras zonas geográficas, como por ejemplo Latinoamérica y América del Norte (Canadá, Estados Unidos). También sería conveniente realizar una comparación sobre esta cuestión en Estados con diferentes modelos de centralización educativa (central, federal, municipal...).

En definitiva, la infancia inmigrante se enfrenta a una red de mecanismos de discriminación en la UE que dificulta notablemente su integración, su ascenso social y el respeto de sus derechos fundamentales, incluyendo el de la educación. Por este motivo, toda la comunidad educativa, desde ámbitos supranacionales a locales han de reconocer su responsabilidad en esta cuestión y actuar éticamente en defensa de la dignidad humana, dejando de lado nacionalismos, miedos y estereotipos artificiales.

\section{Referencias}

Adam, H. (2015) Xenophobia, Asylum Seekers, and Immigration Policies in Germany. Nationalism and Ethnic Politics, 21(4), 446464

Ahmadi, F., Darvishpour, M., Ahmadi, N., y Palm, I. (2020). Diversity barometer: attitude changes in Sweden. Nordic Social Work Research, 10(1), 21-38.

Aris Escarcena, J. P. (2016). ¿La crisis de los refugiados? Consecuencias humanas de las políticas de asilo europeas. Documentación Social, 180, 203-221.

Astier, C., y Errasti, A. (2018). La crisis europea de la política: pluralismo etno-religioso y el auge del populismo y la extrema derecha en Europa. Cuadernos Europeos de Deusto, 59, 27-34.

Baltaci, A. (2017). A comparison of syrian migrant students in Turkey and Germany: Entrepreneurial tendencies and career expectations. European Journal of Educational Research, 6(1), 1527.

Bini, E. (2017). Famiglie migranti con minori disabili. Prospettive inclusive nel sistema socio-sanitario e scolastico italiano. Culture e Studi del Sociale, 2(2), 203-211.

Campani, G. (2019). La migración europea y la crisis de los refugiados: un proceso complejo y multifacético. Collectivus, Revista de Ciencias Sociales, 6(1), 15-34.

Carta de los derechos fundamentales de la Unión Europea. Diario Oficial, serie C, número 202/02, de 7 de junio de 2016.

Convención Internacional sobre la Eliminación de todas las Formas de Discriminación Racial, 21 de diciembre, 1965.

Convención sobre el Estatuto de los Refugiados, 28 de julio, 1951.

Convención sobre la Eliminación de todas las formas de Discriminación contra la Mujer, 18 de diciembre, 1979.

Convención sobre los Derechos del Niño, 20 de noviembre, 1989.
Corchia, L. (2015). Le seconde generazioni di stranieri in Italia. En M. Aldo Toscano y A. Cirillo (Eds.), XENIA. Nueve pe l'integrazione sociale (pp. 85-99). FrancoAngeli.

De Beco, G. (2009). Right to Education Indicator based on the $4 \mathrm{~A}$ framework Concept Paper. Right to Education Initiative.

Declaración de los Derechos del Niño, 20 de noviembre, 1959.

Declaración de Nueva York para los Refugiados y los Migrantes, 3 de octubre, 2016.

Declaración Universal de los Derechos Humanos, 10 de diciembre, 1948.

Edling, S., y Francia, G. (2017). Newly arrived pupils and violence: A CDA analysis of political advices regarding strategies and responsibilities for various actors in Swedish education. Education Sciences \& Society-Open Access Journal, 8(1), 137-153.

Etxeberria, F. y Elosegui, K. (2010). Integración del alumnado inmigrante: obstáculos y propuestas. Revista Española de Educación Comparada, 16, 235-263.

Eurostat. (2020). Estadísticas de asilo.

Eurydice. (2019). Integración del alumnado de procedencia migrante. Oficina de Publicaciones de la Unión Europea.

Eurydice. (2020). The structure of the European education systems 2020/21. Schematic Diagrams. Oficina de Publicaciones de la Unión Europea

Ferrero-Turrión, R. (2016). Europa sin rumbo. El fracaso de la UE en la gestión de la crisis de refugiados. Revista de Estudios Internacionales Mediterráneos, 21, 159-176.

Flores González, B. (2018). La protección jurídica de los menores inmigrantes no acompañados en España. Revista de Derecho Civil, 5(2), 321-362.

García Ruiz, M. (2015). De la quiebra del Lehman Brothers a la crisis de la deuda soberana en Europa: el quinquenio gris de los 
mercados financieros internacionales. Economía y Desarrollo, 154(1), 45-59.

García Segura, C. (2019). La representación exterior de la Unión Europea en un contexto de crisis. En F. Guirao, y J. Pich, ¿Una Unión Europea en crisis? Reflexiones para un debate urgente (pp. 145-167). Catarata.

González Begega, S. y Guillén Rodríguez, A. M. (2018). Austeridad y reforma del bienestar en la Europa del Sur. Transformación y heterogeneidad institucional. Papeles de Europa, 31(1), 69-79.

Grabbe, C. (2018). Education policy and Integration in Germany. LoGoSO Research Papers, 1, 1-18.

Herzog, B. (2011). Exclusión discursiva. Hacia un nuevo concepto de la exclusión social. Revista Internacional de Sociología, 69(3), 607-626.

Horvat, S., y Žižek, S. (2014). El sur pide la palabra. Sin fronteras.

Izaola, A., y Zubero, I. (2015). La cuestión del otro: forasteros, extranjeros, extraños y monstruos. Papers: Revista de Sociologia, 100(1), 105-129.

Koehler, C. (2017). Continuity of learning for newly arrived refugee children in Europe. NESET II ad hoc question, 2, 1-33.

León, M., y Subirats, J. (2015). Estados de bienestar que hacen aguas. En P. Morillas, E. Sánchez-Montijano y E. Soler (Coords.), Europa ante la crisis de los refugiados 10 efectos colaterales (pp. 11-12).

López Ortega, A. I. (2017). La explotación del conflicto multicultural por la nueva extrema derecha valenciada. Nómadas. Revista Crítica de Ciencias Sociales y Jurídicas, 3, 121-133.

López Pereira, J. L. (2018). El sistema público de pensiones en España, Grecia e Italia durante la gran recesión de 2008. Análisis en perspectiva comparada de reformas y efectos. Revista Cuadernos Manuel Giménez Abad, 16, 132-152.

Modesti, M. (2011). I figli dei migranti ei diritti umani. En V. Maher (Ed.), Antropologia e diritti umani nel mondo contemporaneo (pp. 149-161). Rosenberg \& Sellier.

Neubauer Esteban, A. (2020). El derecho a la educación de los menores refugiados y solicitantes de asilo desde el enfoque basado en los derechos humanos: dificultades, finalidad e intervención educativa. Revista Española de Educación Comparada, 35, 70-91.

Nilsson, J., y Bunar N. (2016). Educational Responses to Newly Arrived Students in Sweden: Understanding the Structure and Influence of PostMigration Ecology. Scandinavian Journal of Educational Research, 60(4), 399-416.

Ocampo Gómez, C. I., y Cid Souto, B. (2012). Formación, experiencia docente y actitudes de los profesores de infantil y primaria ante la educación escolar de hijos de personas inmigrantes en España. Revista de Investigación Educativa, 30(1), 111-130.

Pacto Mundial para una Migración Segura, Ordenada y Regular, 13 de julio, 2018.

Parlamento Europeo. (2019). Resultados de las elecciones europeas de 2019.

Payero López, L. (2019). La Unión Europea ante la gestión de la crisis de los refugiados. Cuadernos Electrónicos de Filosofía del Derecho, 37(27), 134-156.

Pelé Illie, A. (2004). Una aproximación al concepto de dignidad humana. Universitas: Revista de filosofía, derecho y política, 1, 913.

Pou, V. (2018). Hacia el relanzamiento de la Unión Europea. El Brexit y Trump como revulsivos. Milenio Publicaciones.
Prevert, A., Navarro Carrascal, O., y Bogalska-Martin, E. (2012). La discriminación social desde una perspectiva psicosociológica. Revista de Psicología, 4(1), 7-20.

Puig Rovira, J. M., Gijón Casares, M., Martín García, X., Rubio Serrano, L. (2011). Aprendizaje-servicio y Educación para la Ciudadanía. Revista de Educación, 1, 45-67.

Rabossi, E. (1990). Derechos Humanos: El principio de igualdad y la discriminación. Revista del Centro de Estudios Constitucionales, (7), 175-192.

Rahona López, M., y Morales Sequera, S. (2013). Diferencias en el rendimiento educativo de nativos e inmigrantes en España. Revista de Sociología de la Educación (RASE), 6(1), 72-90.

Reath, W. (2013). Mother Tongue Tuition in Sweden - Curriculum Analysis and Classroom Experience. International Electronic Journal of Elementary Education, 6(1), 95-116.

Recomendación del Consejo de 9 de diciembre de 2013 relativa a la adopción de medidas eficaces de integración de los gitanos en los Estados miembros. (2013/C 378/01). Diario Oficial, serie C, número 378/1, de 24 de diciembre de 2013.

Reglamento no 233/2014 Del Parlamento Europeo y del Consejo de 11 de marzo de 2014 por el que se establece un Instrumento de Financiación de la Cooperación al Desarrollo para el período 20142020. Diario Oficial, serie L, número 77/44, de 15 de marzo de 2014.

Rubiés, J. (2019). La Unión Europea y el proceso del Brexit. En F. Guirao, y J. Pich, ¿Una Unión Europea en crisis? Reflexiones para un debate urgente (pp. 168-200). Catarata.

Sanahuja, J. A. (2016). La Unión Europea y la crisis de los refugiados: fallas de gobernanza, securitización y "diplomacia de chequera". En M. Mesa (Coord.), Retos inaplazables en el sistema internacional Anuario 2015-2016 (pp. 71-105).

Santagasti, M. (2016). Interculturalism, education and society: Education policies for inmigrant students in Italy. Australian \& New Zealand Journal of European Studies, 8(2), 6-20.

Solís, P. (2017). Discriminación estructural y desigualdad social. Consejo Nacional para Prevenir la Discriminación.

Soriano-Ayala, E., y González-Jiménez, A. J. (2010). El poder educativo de las asociaciones de inmigrantes en las escuelas multiculturales. RELIEVE. Revista Electrónica de Investigación y Evaluación Educativa, 16(1), 1-20.

UNICEF (2008). Un enfoque de la educación para todos basado en los derechos humanos. https://bit.ly/2Txr0Jh

Valle López, J. M., y Matarranz García, M. (2015). La Unión Europea: un protagonismo de excepción en la pedagogía del siglo XXI. Padres Y Maestros, 363, 68-72. 